\title{
Illumination conditions at the lunar poles: Implications for future exploration
}

\author{
P. Gläser ${ }^{\mathrm{a}, *}, J$ Oberst $^{\mathrm{a}, \mathrm{b}, \mathrm{c}}$, G. A. Neumann ${ }^{\mathrm{d}}$, E. Mazarico ${ }^{\mathrm{d}}$, E.J. Speyerer ${ }^{\mathrm{e}}$, M. S. \\ Robinson ${ }^{\mathrm{e}}$ \\ ${ }^{a}$ Technische Universität Berlin, Institute of Geodesy and Geoinformation Science, 10623 Berlin, \\ Germany \\ ${ }^{b}$ German Aerospace Center, Institute of Planetary Research, 12489 Berlin, Germany \\ ${ }^{c}$ Extraterrestrial Laboratory, Moscow State University for Geodesy and Cartography, \\ RU-105064 Moscow, Russia \\ ${ }^{d}$ NASA Goddard Space Flight Center, Code 698, Greenbelt, MD 20771, USA \\ ${ }^{e}$ Arizona State University, School of Earth and Space Exploration, Tempe, AZ 85287, USA
}

\begin{abstract}
We produced 400 x $400 \mathrm{~km}$ Digital Terrain Models (DTMs) of the lunar poles from Lunar Orbiter Laser Altimeter (LOLA) ranging measurements. To achieve consistent, high-resolution DTMs of $20 \mathrm{~m} /$ pixel the individual ranging profiles were adjusted to remove small track-to-track offsets. We used these LOLADTMs to simulate illumination conditions at surface level for $50 \times 50 \mathrm{~km}$ regions centered on the poles. Illumination was derived in one-hour increments from 01 January, 2017 to 01 January, 2037 to cover the lunar precessional cycle of 18.6 years and to determine illumination conditions over several future mission cycles. We identified three regions receiving high levels of illumination at each pole, e.g. the equator-facing crater rims of Hinshelwood, Peary and Whipple for the north pole and the rim of Shackleton crater, and two locations on a ridge between Shackleton and de Gerlache crater for the south pole. Their average illumination levels range from $69.5 \%$ to $82.9 \%$, with the highest illumination levels found at the north pole on the rim of Whipple crater. A more detailed study was carried out for these sites as targets for a lander and/or rover equipped with solar arrays. For this purpose we assumed a lander with a structural height of two meters above the ground (height of the solar panels). Here average illumination levels range from $77.1 \%$ to $88.0 \%$, with the maximum found at the ridge between Shackleton and de Gerlache crater on the south pole. Distances, sizes and slopes of nearby Permanently Shadowed Regions (PSRs) as a prime science target were also assessed in this case.
\end{abstract}

Keywords: polar illumination; Moon; landing sites; space exploration; LOLA 


\section{Introduction}

The lunar day lasts about 29.5 Earth-days, whereby the equatorial region of the Moon is illuminated for about 15 Earth-days followed by a 15 Earth-day long night. Near the poles, however, illumination is more complex and no specific statement about the duration of illumination periods can be made. As a consequence of the small $1.54^{\circ}$ rotational obliquity, illumination in polar regions differs considerably from that of regions at lower latitudes. The interaction of grazing sunlight and large local topography variations leads to extreme illumination conditions. Permanently Shadowed Regions (PSRs) exist in close proximity to areas receiving illumination for the greater part of the year with only short periods in darkness of typically just a few days (Mazarico et al., 2011; Speyerer and Robinson, 2013; Gläser et al., 2014). Accordingly, PSRs are amongst the coldest areas on the Moon (Paige et al., 2010) and have long been believed to harbor enhanced concentrations of volatiles and hence possibly also water-ice (Watson et al., 1961; Feldman et al., 2001; Mitrofanov et al., 2012).

For future lunar missions, it can be assumed, that rovers, landers or astronauts shall explore the lunar surface for longer time-scales than just a few days as was the case during the Apollo era. The Mars Exploration Rover Opportunity, for instance, has already traversed the Martian surface for more than twelve years and has traveled more than forty kilometers only relying on solar power (Arvidson et al., 2015). Landing at locations with extended illumination and within reach of PSRs, a prime science exploration target (Cohen et al., 2015), a mission designed with traversing capabilities could repeatedly enter PSRs and recharge close-by in sunlit areas. PSRs are proposed to harbor water-ice concentrations (Watson et al., 1961), of which some were recently identified to be spots of water-rich permafrost (Mitrofanov et al., 2012; Spudis et al., 2013; Miller et al., 2014). Especially, the north polar craters Peary and Whipple were found to be Neutron Suppression Regions (NSR) as measured by the Lunar Reconnaissance Orbiter (LRO) Lunar Exploration Neutron Detector (LEND). NSRs are regions with enhanced hydrogen content, which combined with the low temperatures

*Corresponding author: Philipp Gläser, Technische Universität Berlin, Institute of Geodesy and Geoinformation Science, Str. des 17. Juni 135, 10623 Berlin, Germany; Phone, +49 (0)30 314-28828

Email address: philipp.glaeser@tu-berlin.de (P. Gläser) Preprint submitted to PSS

June 29, 2017 
inside some of the PSRs make them possible locations for water-rich lunar permafrost (Mitrofanov et al., 2012). The local water-ice resources encountered in these PSRs could be used for the production of fuel in addition to the recovery of drinking water.

Several techniques are suitable to identify PSRs and regions of enhanced illumination. For instance, Bussey et al. (1999) and Speyerer and Robinson (2013) used lunar imagery for their studies. Margot et al. (1999) used Digital Terrain Models (DTMs) of the lunar poles derived from Earth-based radar observations. DTMs are advantageous because illumination conditions can be simulated over very large time scales and are generally independent of time (e.g. an image shows the instantaneous illumination for one specific time). Noda et al. (2008), Bussey et al. (2010), Mazarico et al. (2011), McGovern et al. (2013), De Rosa et al. (2012) and Gläser et al. (2014) all used laser-derived DTMs with ever increasing resolutions from $470 \mathrm{~m} /$ pixel to $20 \mathrm{~m} /$ pixel. A technique to infer illumination based on DTMs is shown in, e.g. Mazarico et al. (2011).

This study investigates illumination conditions at the north pole in greater detail than previous studies and reveals areas of extensive illumination. This work complements and also updates Gläser et al. (2014). The analysis is based upon 20 m/pixel DTMs derived from Lunar Orbiter Laser Altimeter (LOLA) (Smith et al., 2010) ranging measurements. The focus of this work lies on a 50 x $50 \mathrm{~km}$ area centered at each pole.

\section{Data}

For this study precise, large-scale and high-resolution LOLA-DTMs are needed for both poles. LOLA is the laser altimeter onboard LRO, which was launched in 2009 and is still operational. At the laser pulse rate of $28 \mathrm{~Hz}$, along- and acrosstrack spacing of the spots is just 10-12 $\mathrm{m}$. The nominal ranging precision of the instrument is $10 \mathrm{~cm}$ and its vertical accuracy is $<1 \mathrm{~m}$ (Smith et al., 2010, 2017). In Gläser et al. (2014) a south-polar LOLA-DTM was already created using coregistration techniques, which has been updated for this work. Accordingly, 400 x $400 \mathrm{~km}$ polar LOLA-DTMs sampled at $20 \mathrm{~m} /$ pixel were generated for this work using the most recent LOLA data (up to LRO_ES_48 - July 2016). For the generation of the south-polar LOLA-DTM, single LOLA tracks were first coregistered to a Narrow Angle Camera (NAC) DTM. For the north-polar DTM, however, no appropriate NAC-DTM was available near the study area and hence was exclusively processed using LOLA data. The initial DTM was chosen to be a small-scale LOLA DTM centered at the north pole from which successively larger areas were derived through co-registration in an iterative way (Gläser et al., 
2013, 2014).
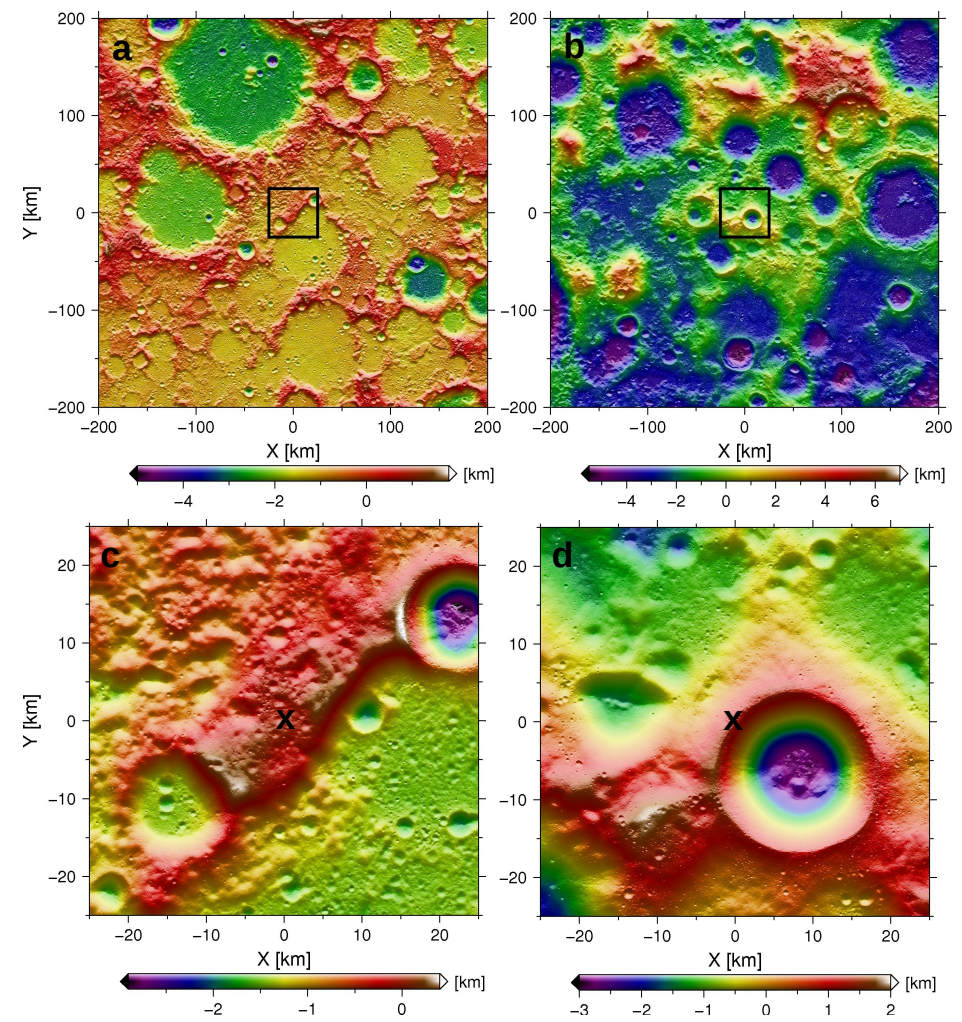

Figure 1: Shaded and height color-coded 400 x $400 \mathrm{~km}$ LOLA-DTM with $20 \mathrm{~m} /$ pixel resolution showing topography centered at the lunar pole. The area of interest for which illumination conditions are investigated is marked by the black square. (a) North pole. (b) South pole. (c) Inset of the $50 \times 50 \mathrm{~km}$ area of interest at the north pole, black cross in the center marks the position of the pole. (d) Inset of the $50 \times 50 \mathrm{~km}$ area of interest at the south pole, black cross in the center marks the position of the pole. All units are in kilometers and maps are displayed in stereographic projection.

The LOLA data set contains 10,240 individual tracks above $60^{\circ} \mathrm{N}$ and 28,629 tracks above of $60^{\circ} \mathrm{S}$. The vast difference in the amount of tracks is due to LRO's current (and initial, during commissioning) elliptical orbit with its periselene near the south pole. Consequently, the aposelene is located over the lunar north pole with a distance to the surface too large for the LOLA instrument to perform range measurements (Smith et al., 2017). During the nominal mapping orbit LRO was in a circular orbit from which it was possible to range globally. For the generation of our polar DTMs $\left(>\sim 83^{\circ} \mathrm{N} / \mathrm{S}\right)$, only co-registered tracks meeting certain quality criteria were used in the processing (for more informa- 
tion about co-registration quality criteria please see (Gläser et al., 2013)). Finally 6,281 co-registered tracks consisting of 84,876,446 LOLA surface-spots make up the north-polar DTM and 12,179 co-registered tracks containing 161,138,573 spots were processed to create the south-polar DTM (Fig. 1a,b).

The $50 \times 50 \mathrm{~km}$ area of interest centered at the north pole (Fig. 1c) contains $6,250,00020 \times 20 \mathrm{~m}$ sized pixels, of which 4,178,695 pixels are filled with between 1-12 LOLA-spots (in total the DTM contains 7,256,206 spots). Consequently, $67 \%$ of the pixels in Fig. 1c are created from actual LOLA-spots and $33 \%$ are filled by interpolation. To assess the error introduced by the interpolation using the Generic Mapping Tool (GMT) surface routine, every third pixel in the DTM was deleted and then interpolated again (Wessel et al., 2013; Smith and Wessel, 1990). The resulting height residuals are normal distributed around zero and have a standard deviation of ten centimeters. Most height differences occur at steeper regions within the DTM, such as along the walls of larger craters of which some reach the one meter level. The rather small errors due to the interpolation can be explained by self-affine lunar topography and the fact that the used interpolation technique assumes a positively autocorrelated topography function. At the poles, which are part of the lunar highlands, Hurst exponents above 0.95 are typical. A Hurst exponent of 1 implies self-similar topography where the roughness at small scales is exactly replicated at large scales (Rosenburg et al., 2011).

Similarly, the area of interest centered at the south pole (Fig. 1d) also contains $6,250,00020 \times 20 \mathrm{~m}$ sized pixels, of which 4,835,123 pixels are filled with between 1-16 LOLA-spots (in total the DTM contains 9,855,592 spots). Hence, $77 \%$ of the pixels in Fig. 1d are created from actual LOLA-spots and 23\% are filled by interpolation. The 400 x $400 \mathrm{~km}$ south-polar DTM shown in Fig. 1b shows an increase of $9 \%$ of pixels filled by at least one LOLA spot when compared to the version presented in Gläser et al. (2014).

For the displayed areas in Fig. 1c,d a horizon map data-base was derived, which is needed to simulate illumination (Mazarico et al., 2011). Horizon maps describe the visible horizon line as seen from each pixel in the DTM. An example of such a horizon line seen by an observer located directly at the north pole is shown in Fig. 2. Once the horizon line of a specific location on the Moon is known it is straight forward to derive illumination. Here, the azimuth, elevation and size of the solar disk at a certain time is compared to the horizon line and the fraction of the visible solar disk can be determined (Mazarico et al., 2011; Gläser et al., 2014). From the comparison of horizon maps with the location of the Sun over many time-steps average illumination values can be derived for 
each location. Average illumination is calculated by summing up and averaging all visible fractions of the solar disk, which results in a numeric value between zero (Sun was never visible) and 1 (Sun was always fully visible). However, a value of 0.5 , for instance, could mean half of the solar disk was visible the whole time or the Sun was fully visible for half the time and not visible the other half. In reality the distribution of visible solar disk fractions will be more complex and hence average illumination is a good indicator of how intensely a location is illuminated but not necessarily how long in time.
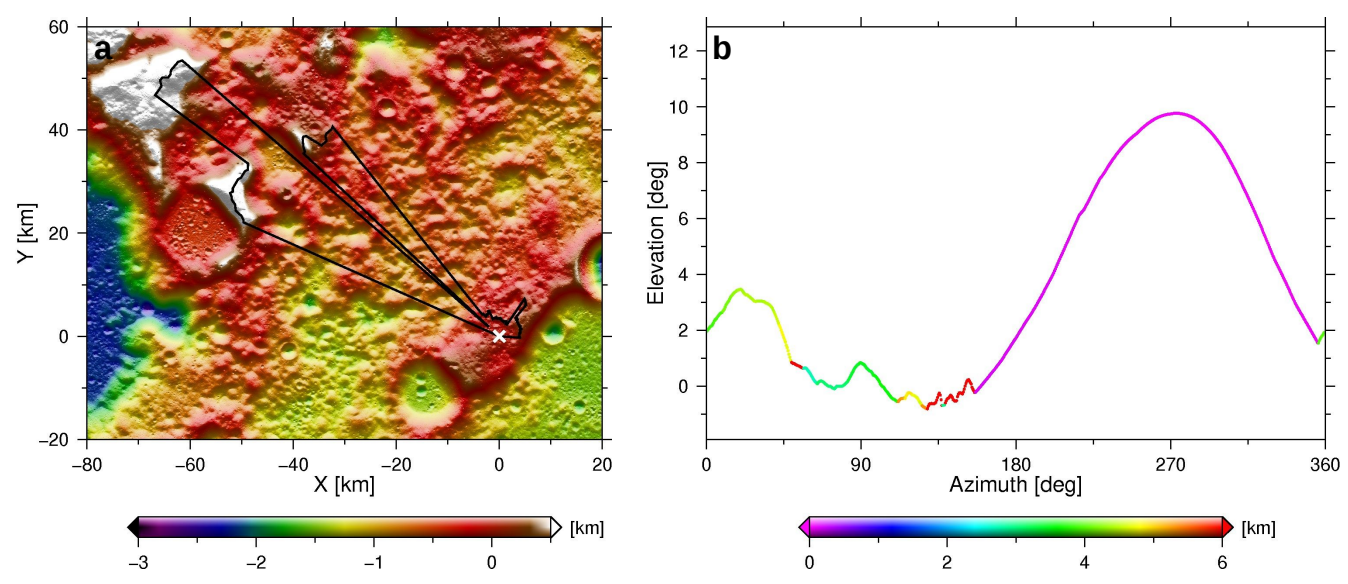

Figure 2: Plot of the horizon as seen by an observer at the north pole at surface level. (a) Horizon line plotted on the DTM, where the x-axis indicates $0^{\circ}$ azimuth counting counter-clockwise. The north pole is marked with a white $\mathrm{x}$. (b) The horizon is displayed in degrees above the local plane (elevation) along the azimuth. The horizon line is color-coded by the distance to the obstructing topography (horizon). Note: the maximum elevation of the Sun at the poles is $1.54^{\circ}$.

\section{Results}

Illumination at the poles depends not only on local topography and the slight seasons caused by the $1.54^{\circ}$ tilt, but also on the height above ground and on the lunar precessional cycle. In order to specify regions that are favorable landing sites over larger time scales, we simulated illumination near the poles over a twenty-year period (01 January, 2017 to 01 January, 2037). This period covers a full lunar precessional cycle of 18.6 years and all seasonal and orbital illumination effects. Further we investigated illumination at surface level and at two meters above ground. For the classification as a potential landing sites at each pole, we first identified the regions with highest average illumination at surface level. We then identified clusters of high average illumination at two meters 
above ground at each site, which are most favorable for power generation by solar panels. Further, we identified PSRs within each region and evaluated the shortest distance to the potential landing site clusters. Here, the establishment of a hybrid landing site for equipment supporting a robotic and/or human mission would be most viable. Although most of the sites we investigated in detail have been reported in previous studies our resolution is higher than in all previous image and DTM-based studies (Bussey et al., 1999; Noda et al., 2008; Bussey et al., 2010; Mazarico et al., 2011; De Rosa et al., 2012; Speyerer and Robinson, 2013). Further, the temporal resolution by simulating illumination on DTMs is superior than any image-based approach.

Illumination at surface level. First, we investigated polar illumination conditions at surface level and assembled average illumination maps (Fig. 3a,b). These maps display average illumination over the twenty-year period sampled each hour. The maps also show three regions at each pole that contain locations with distinctively higher average illumination levels $(>\sim 70 \%)$ than the surroundings. At the north pole the three regions are all located on equator-facing crater rims, namely of Hinshelwood, Peary and Whipple craters. To assess the robustness of the identified locations the resolution of the DTM was altered and illumination was re-calculated for the case of Whipple crater. As expected, these sites are persistent when evaluating at a coarser resolution of forty meters where the calculated illumination is generally even higher due to the smoothing of the horizon (Gläser et al., 2014). But also on a DTM with a resolution of ten meters the locations of high average illumination persist and confirm the previous findings. Near the south pole one region is located on the rim of Shackleton crater and two are found on a ridge between the Shackleton and de Gerlache craters, referred to as 'Connecting Ridge C1 and C2' (De Rosa et al., 2012). The location referred to as Shackleton region in this study comprises the two regions called SR1 and SR2 in Gläser et al. (2014). Further the location referred to as Connecting Ridge C1 is the same as the one studied in Gläser et al. (2014) and the location Connecting Ridge $\mathrm{C} 2$ is a newly discussed region. The identified locations form rather thin and elongated structures up to several kilometers along the rims and ridges, whose width however, only reaches twenty to forty meters (one to two pixels). The maximum average illumination of $82.9 \%$ is found near the north pole along the rim of Whipple crater, revealing the site with the most intense insolation. It also happens to be the polar location with the longest solar visibility, describing the percentage of a given period in which any portion of the Sun is visible. On average, $88.5 \%$ of the time the Sun is at least partially visible there. Further, at this site a lander would also be exposed to the shortest maxi- 
mum time in shadow of just four days and five hours. For each site discussed in this study the reported locations of maximum illumination and, if provided, the maximum time in shadow from recent previous studies (Mazarico et al., 2011; Speyerer and Robinson, 2013) are shown in Table 1. Only Connecting Ridge C2 was not previously reported in the literature and therefore no comparisons can be shown. It can be noted that the results for maximum average illumination from this study are always in between the two previous results, where Mazarico et al. (2011) used a 240 meter per pixel LOLA DTM and Speyerer and Robinson (2013) used 100 meter per pixel LRO Wide Angle Camera (WAC) images. The reason for the difference is that Mazarico et al. (2011) overestimates illumination compared to this study due to smoothing of the close-range topography by the larger grid size. The effect of DTM resolution on the resulting illumination is discussed in Gläser et al. (2014). As mentioned earlier, the identified locations of high average illumination are only twenty to forty meters wide. Hence, the lower resolution of the images used in the study of Speyerer and Robinson (2013) compared to the LOLA DTM of this study is underestimating illumination since small-scale illuminated areas less than 100 meters across might not be detected by WAC. Further, Speyerer and Robinson (2013) only determined the average illumination over a one-year period where orbital and seasonal effects on illumination are not well covered. Solar visibility is only reported in Mazarico et al. (2011) and fits well to our findings given the difference in the resolution of the used LOLA DTMs. Nevertheless Mazarico et al. (2011) finds the longest solar visibility on the CR1 site near the south pole which is only the third best site in this study. Maximum time in shadow is an important parameter regarding the time a rover is exposed to very low temperature levels while there is no possibility to charge the batteries. Beside for the south polar site CR1, this parameter is only shown in Speyerer and Robinson (2013). Due to the higher temporal and spatial resolution of this study the found maximum times in shadow are found to be up to seventy percent shorter than reported in Speyerer and Robinson (2013). Only at Shackleton crater the reported time in shadow for the most illuminated spot is shorter in the image-based approach than in this study.

Also note that the location of maximum average illumination reported in Gläser et al. (2014) is located at a different location than in this study. Although both are located on the rim of Shackleton crater they are roughly three kilometers apart and differ by about five percent in illumination. This is due to the improved topography of the LOLA DTM in this study. All six sites are in close proximity to several small (up to one-hundred meters in diameter) to medium-sized (one-hundred meters to one kilometer in diameter) PSRs. Table 1 summarizes 
the findings of the study of illumination conditions over twenty years at surface level and also shows the results from Mazarico et al. (2011) and Speyerer and Robinson (2013).
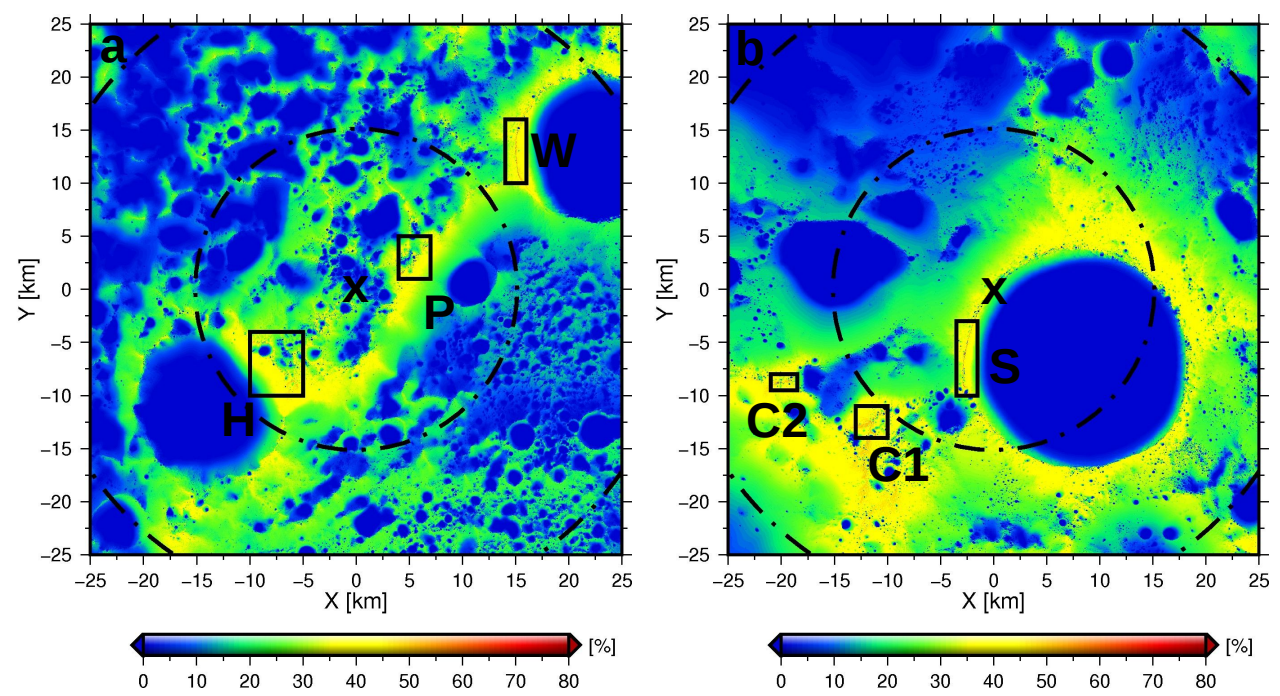

Figure 3: Average illumination (shown from 0-80\%), calculated at surface level for a twenty-year period from January 1, 2017 to January 1, 2037 and sampled hourly. The black crosses indicate the location of the pole, the smaller dashed circle is drawn $0.5^{\circ}$ from the pole, the second circle at $1^{\circ}$. (a) North pole: The three areas with highest average illumination are the equator-facing rims of Hinshelwood (H), Peary (P) and Whipple (W) craters. (b) South pole: The three areas with highest average illumination are Shackleton crater $(\mathrm{S})$ and two regions on Connecting Ridge (C1), (C2). Map axis units are in kilometers and map is displayed in gnomonic projection.

Illumination at two meters above ground level. We assume that future lunar surface missions aiming for either of the poles, will mainly or exclusively rely on solar power. Solar power is virtually the only long-term energy source available for future lunar surface missions. Such solar arrays will be deployed up to several meters above ground, where illumination levels can be substantially higher than at surface level. Locations receiving extended illumination are most attractive because an almost continuous power supply can be guaranteed. Due to the absence of an atmosphere, power management can be assessed with confidence based solely on surface topography and geometry. Illumination conditions at a height of two meters above ground were investigated over the twenty-year period to simulate insolation received by solar panels mounted on a rover or lander. As can be seen in the corresponding average illumination maps, the aforementioned regions of high average illumination at surface level grow substantially in size 


\begin{tabular}{|c|c|c|c|c|c|}
\hline Site & $\begin{array}{r}\text { Latitude } \\
{\left[{ }^{\circ}\right]}\end{array}$ & $\begin{array}{r}\text { Longitude } \\
{\left[{ }^{\circ}\right]}\end{array}$ & $\begin{array}{r}\text { Max. average } \\
\text { illumination } \\
\text { [\%] }\end{array}$ & $\begin{array}{r}\text { Solar } \\
\text { visibility } \\
{[\%]}\end{array}$ & $\begin{array}{r}\text { Max. time } \\
\text { in shadow } \\
{[\mathrm{h}]}\end{array}$ \\
\hline \multicolumn{6}{|l|}{$\begin{array}{r}\text { North pole } \\
\text { Hinshelwood, } \mathrm{H}\end{array}$} \\
\hline This study & 89.6564 & 326.44 & 72.6 & 81.2 & 392 \\
\hline Mazarico et al. (2011) & 89.65 & 326.13 & 80.6 & 86.9 & - \\
\hline Speyerer and Robinson (2013) & 89.660 & 324.1 & 37.0 & - & 650 \\
\hline \multicolumn{6}{|l|}{ Peary, $\mathrm{P}$} \\
\hline This study & 89.7260 & 123.11 & 69.5 & 78.3 & 334 \\
\hline Mazarico et al. (2011) & 89.85 & 110.38 & 78.1 & 85.0 & - \\
\hline Speyerer and Robinson (2013) & 89.723 & 122.8 & 44.7 & - & 445 \\
\hline \multicolumn{6}{|l|}{ Whipple, W } \\
\hline This study & 89.3766 & 126.48 & 82.9 & 88.5 & 101 \\
\hline Mazarico et al. (2011) & 89.37 & 126.80 & 84.6 & 89.4 & - \\
\hline Speyerer and Robinson (2013) & 89.370 & 127.6 & 63.3 & - & 303 \\
\hline \multicolumn{6}{|l|}{$\begin{array}{r}\text { South pole } \\
\text { Connecting Ridge, } \mathrm{C} 1\end{array}$} \\
\hline This study & -89.4505 & 222.84 & 76.0 & 81.3 & 268 \\
\hline Mazarico et al. (2011) & -89.45 & 222.69 & 89.0 & 92.7 & 141.12 \\
\hline Speyerer and Robinson (2013) & -89.418 & 221.3 & 64.2 & - & 308 \\
\hline \multicolumn{6}{|l|}{ Connecting Ridge, $\mathrm{C} 2$} \\
\hline This study & -89.3157 & 245.94 & 75.6 & 80.6 & 166 \\
\hline \multicolumn{6}{|l|}{ Shackleton, S } \\
\hline This study & -89.7849 & 203.97 & 81.0 & 85.5 & 221 \\
\hline Mazarico et al. (2011) & -89.78 & 204.27 & 86.7 & 90.5 & - \\
\hline Speyerer and Robinson (2013) & -89.740 & 201.2 & 71.6 & - & 145 \\
\hline
\end{tabular}

Table 1: List of locations at the lunar poles with high average illumination at surface level averaged over a twenty-year period. Latitude, longitude, solar visibility and longest time in shadow found in this study, Mazarico et al. (2011) and Speyerer and Robinson (2013) are reported for each site if available. Note that this study and the one carried out by Mazarico et al. (2011) are based on a LOLA DTM of 20 and $240 \mathrm{~m} /$ pixel, respectively, and over a twenty year period. Speyerer and Robinson (2013) used 100 m/pixel WAC images over a period of one year.

and intensity when raised two meters above ground (Fig. 4a,b). The locations receiving high average illumination levels now stretch up to several hundred me- 
ters across the crater rims and ridges. The maximum average illumination of $88.0 \%$ is now found at the south pole at Connecting Ridge (C1) (Fig. 4b). There also the longest solar visibility of $92.1 \%$ is found. Note that the locations found at two meters above ground do not coincide with the locations of maximum average illumination at surface level. Also note, that at Peary and Shackleton crater the location of longest solar visibility does not coincide with the location of maximum average illumination. The maximum times in shadow for Hinshelwood, Peary, Connecting Ridge $\mathrm{C} 1$ and Shackleton are up to seventy percent shorter than at surface level. Contrary, Whipple and Connecting Ridge C2 do not benefit from raising the observer by two meters above ground regarding maximum time in shadow. Table 2 summarizes the findings of the study of illumination
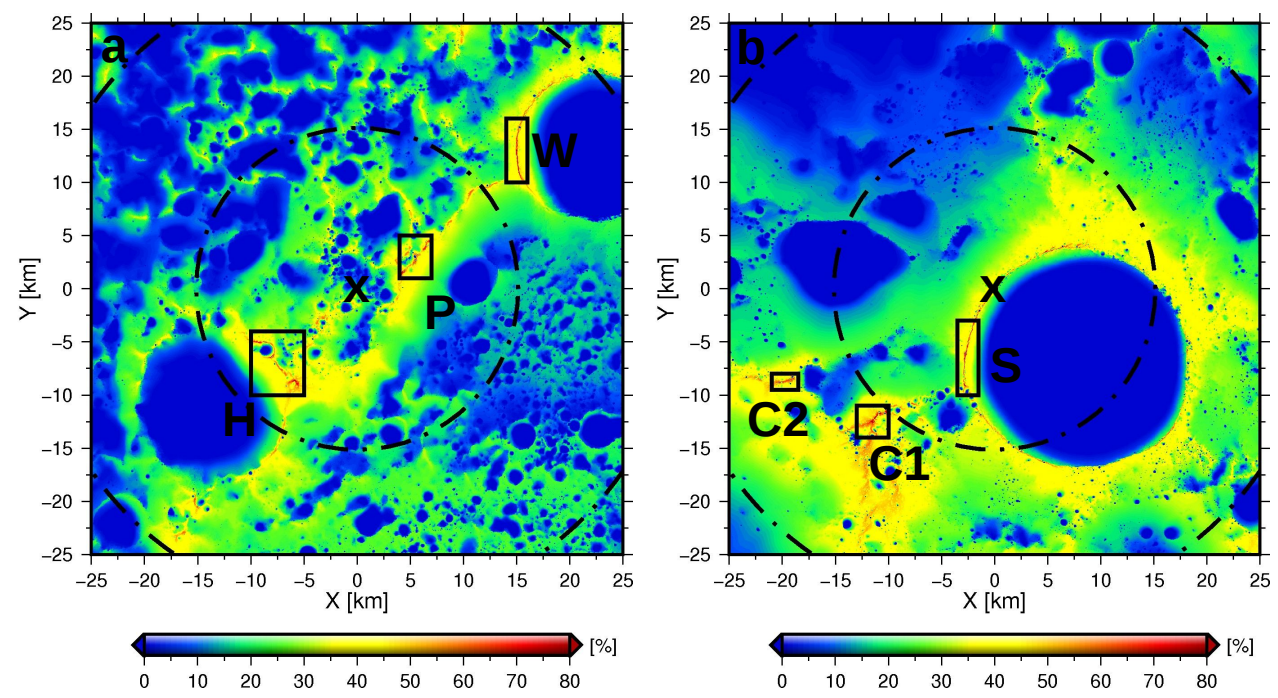

Figure 4: Average illumination (shown from 0-80\%), calculated two meters above ground for a twenty-year period from January 1, 2017 to January 1, 2037 and sampled hourly. The black crosses indicate the location of the pole, the smaller dashed circle is drawn $0.5^{\circ}$ from the pole, the second circle at $1^{\circ}$. (a) North pole: The three areas with highest average illumination are the equator-facing rims of Hinshelwood (H), Peary (P) and Whipple (W) craters. (b) South pole: The three areas with highest average illumination are Shackleton crater (S) and two regions on Connecting Ridge $(\mathrm{C} 1),(\mathrm{C} 2)$. Map axis units are in kilometers and map is displayed in gnomonic projection.

conditions over twenty years at $2 \mathrm{~m}$ above ground. 


\begin{tabular}{|r|r|r|r|r|r|}
\hline Site & $\begin{array}{r}\text { Latitude } \\
{\left[{ }^{\circ}\right]}\end{array}$ & $\begin{array}{r}\text { Longitude } \\
{\left[{ }^{\circ}\right]}\end{array}$ & $\begin{array}{r}\text { Max. average } \\
\text { illumination } \\
{[\%]}\end{array}$ & $\begin{array}{r}\text { Solar } \\
\text { visibility } \\
{[\%]}\end{array}$ & $\begin{array}{r}\text { Max. time } \\
\text { in shadow } \\
{[\mathrm{h}]}\end{array}$ \\
\hline North pole & \multicolumn{5}{|c|}{} \\
\hline Pinshelwood, H & 89.6561 & 326.34 & 81.5 & 89.3 & 203 \\
Peary, P & 89.8521 & 109.80 & 77.1 & 85.06 & 124 \\
Whipple, W & 89.8519 & 110.04 & 77.0 & 85.09 & 124 \\
\hline South pole & 89.3663 & 128.11 & 85.1 & 90.8 & 101 \\
\hline Connecting Ridge, C1 & -89.4398 & 222.83 & 88.0 & 92.1 & 112 \\
Connecting Ridge, C2 & -89.3130 & 245.98 & 78.1 & 82.0 & 165 \\
Shackleton, S & -89.6866 & 197.19 & 85.5 & 89.4 & 66 \\
& -89.7815 & 204.70 & 82.1 & 89.8 & 65 \\
\hline
\end{tabular}

Table 2: List of locations at the lunar poles with high average illumination at two meters above ground averaged over a twenty-year period. Latitude, longitude, solar visibility and maximum time in shadow are given for each site. 
Potential landing sites near PSRs. For each of the six sites near the poles at least one nearby PSR was identified, which lies closest to a cluster of high average illumination. The PSRs of interest were defined as areas where the Sun was not visible over the chosen twenty-year period at surface level and have an extent of at least 1,200 $\mathrm{m}^{2}$ (three pixels). Slopes encountered within the PSRs are reported, which are a good approximation of the maximum expected slopes needed to be traversed by a rover from nearby terrain. Water-ice within PSRs is stable as far as ten degrees latitude from the poles. Even if the PSR is subject to nearby stray light and subsequent heating, water-ice in unshaded regions within two degrees latitude is stable in the subsurface (Vasavada et al., 1999). Hence, it can be expected to find surficial water-ice which may be only covered by a very thin regolith layer. In figures 5 and 6 average illumination at two meters above ground is shown for each of the six sites. Here, PSRs (at surface level) are represented in white and locations with average illumination above or equal $70 \%$ are shown in black.

On the rim of north-polar Hinshelwood crater, we identified three clusters of high illumination (H0, H1, H2) and for each one the closest PSR was found (Fig. 5a). The maximum average illumination at each cluster is $81.5 \%, 74.1 \%, 75.0 \%$ and the longest solar visibilities are $89.3 \%, 81.1 \%, 82.3 \%$ for $\mathrm{H} 0, \mathrm{H} 1$ and $\mathrm{H} 2$ respectively. The extent of the cluster at $\mathrm{HO}$ is $82,400 \mathrm{~m}^{2}$ and is almost a continuously connected area. The nearby PSR located 680 meters away amounts to $5,200 \mathrm{~m}^{2}$, where an average slope of $8.2^{\circ}$ and a maximum slope of $11.9^{\circ}$ can be found. Further west along the rim of Hinshelwood the two sections of the cluster H1 appear, situated on a superimposed crater rim. In total the cluster covers 19,600 $\mathrm{m}^{2}$ with the two almost identically sized sections adjoining a $1,200 \mathrm{~m}^{2}$ sized PSR in one hundred meter distance with an average and maximum slope of $2.7^{\circ}$ and $3.5^{\circ}$. The last cluster, $\mathrm{H} 2$, is situated diametrically opposite to $\mathrm{H} 1$ on the other side of the superimposed crater. The $36,800 \mathrm{~m}^{2}$ area is 520 meters away from the closest and rather large PSR of $320,400 \mathrm{~m}^{2}$ with an average slope of $15.8^{\circ}$ and a maximum slope of $22.1^{\circ}$.

Situated on the rim of Peary crater also three clusters of high illumination (P0, $\mathrm{P} 1, \mathrm{P} 2)$ were found with maximum average illumination of $77.1 \%, 72.7 \%, 74.7 \%$ and longest solar visibilities of $85.1 \%, 80.3 \%, 82.2 \%$ respectively (Fig. 5b). The areal extent of the cluster P0 amounts to $36,000 \mathrm{~m}^{2}$ and is only 130 meters away from the nearby $1,200 \mathrm{~m}^{2}$ PSR where an average slope of $4^{\circ}$ and a maximum slope of $5.5^{\circ}$ can be found. Following the rim southwards, the $10,400 \mathrm{~m}^{2}$ cluster of P1 surrounded by three PSRs with a total size of $34,000 \mathrm{~m}^{2}$ emerges. Within the PSRs an average slope of $8.9^{\circ}$ and a maximum slope of $15.1^{\circ}$ are encoun- 
tered. All three PSRs are almost in equal distance to the cluster with a minimum of sixty meters. P2 consists of two clusters of a total of $26,800 \mathrm{~m}^{2}$ with a 3,600 $\mathrm{m}^{2}$ PSR in 310 meters distance. Here, the average slope is $5.1^{\circ}$ and the maximum slope is $9.7^{\circ}$.

On the rim of Whipple crater two rather long clusters W0 and W1 of $175,600 \mathrm{~m}^{2}$ and $48,800 \mathrm{~m}^{2}$ were identified with maximum average illumination of $85.1 \%$ and $76.9 \%$ and maximum solar visibilities of $90.8 \%$ and $83.1 \%$ (Fig. 5c). For W0 the nearby PSR is only $40 \mathrm{~m}$ away and has a size of $1,200 \mathrm{~m}^{2}$ with an average slope of $4.3^{\circ}$ and a maximum slope of $7.0^{\circ}$. It is located in the upper half of the cluster on the side of the rim facing away from Whipple crater. The larger PSR closest to W1 spans $14,400 \mathrm{~m}^{2}$ and is $350 \mathrm{~m}$ away with an average and maximum slope of $12.5^{\circ}$ and $20.0^{\circ}$.

Beside the maximum slope encountered at Hinshelwood $\mathrm{H} 2$, all slopes are below the maximum angle of $21.8^{\circ}$ inclination that a lunar rover can pass (Sheng and Wei-Dong, 2014). The results for the north-polar sites are summarized in Table 3.

The three chosen sites near the south pole are displayed in Fig. 6. At $\mathrm{C} 1$ along Connecting Ridge two clusters can be identified, C1-0 and C1-1, with maximum average illumination levels of $88.0 \%$ and $81.3 \%$ and longest solar visibilities of $92.1 \%$ and $86 \%$ (Fig. 6a). The cluster C1-0 spans an area of $135,200 \mathrm{~m}^{2}$ of almost continuously connected spots of high average illumination with a 5,200 $\mathrm{m}^{2}$ sized PSR in one hundred meters distance. The average slope within the nearby PSR is $8.4^{\circ}$ and the maximum encountered slope is $12.8^{\circ}$. The second cluster, C1-1, spans $26,800 \mathrm{~m}^{2}$ just seventy meters next to a $9,600 \mathrm{~m}^{2}$ sized PSR with an average and maximum slope of $9.2^{\circ}$ and $13.7^{\circ}$, respectively. Previous rover simulations showed that a small amount of mobility in this region could keep the rover illuminated for $94.4 \%$ of the year with the longest eclipse lasting 101 hours (Speyerer et al., 2016).

Further along the ridge, C2 is found. Here, two clusters, C2-0 and C2-1, exist (Fig. 6b). The maximum average illumination levels are $71.6 \%$ and $78.1 \%$ and the longest solar visibility is found to be $75.1 \%$ and $82.0 \%$ respectively. C2-0 spans $4,400 \mathrm{~m}^{2}$ next to an almost equally sized $5,600 \mathrm{~m}^{2}$ large PSR in sixty meters distance. Here, the average slope is $8.4^{\circ}$ and the maximum slope is $11.5^{\circ}$. The $11,600 \mathrm{~m}^{2}$ cluster of C2-1 is found eighty meters next to a $22,800 \mathrm{~m}^{2}$ PSR with an average and maximum slope of $12.1^{\circ}$ and $17.1^{\circ}$.

The last site is located on the rim of Shackleton crater, where we identified three clusters, S0, S1 and S2. The clusters have a maximum average illumination of $85.5 \%, 84.3 \%$ and $73.9 \%$ with longest solar visibilities of $89.4 \%, 89.8 \%$ and 

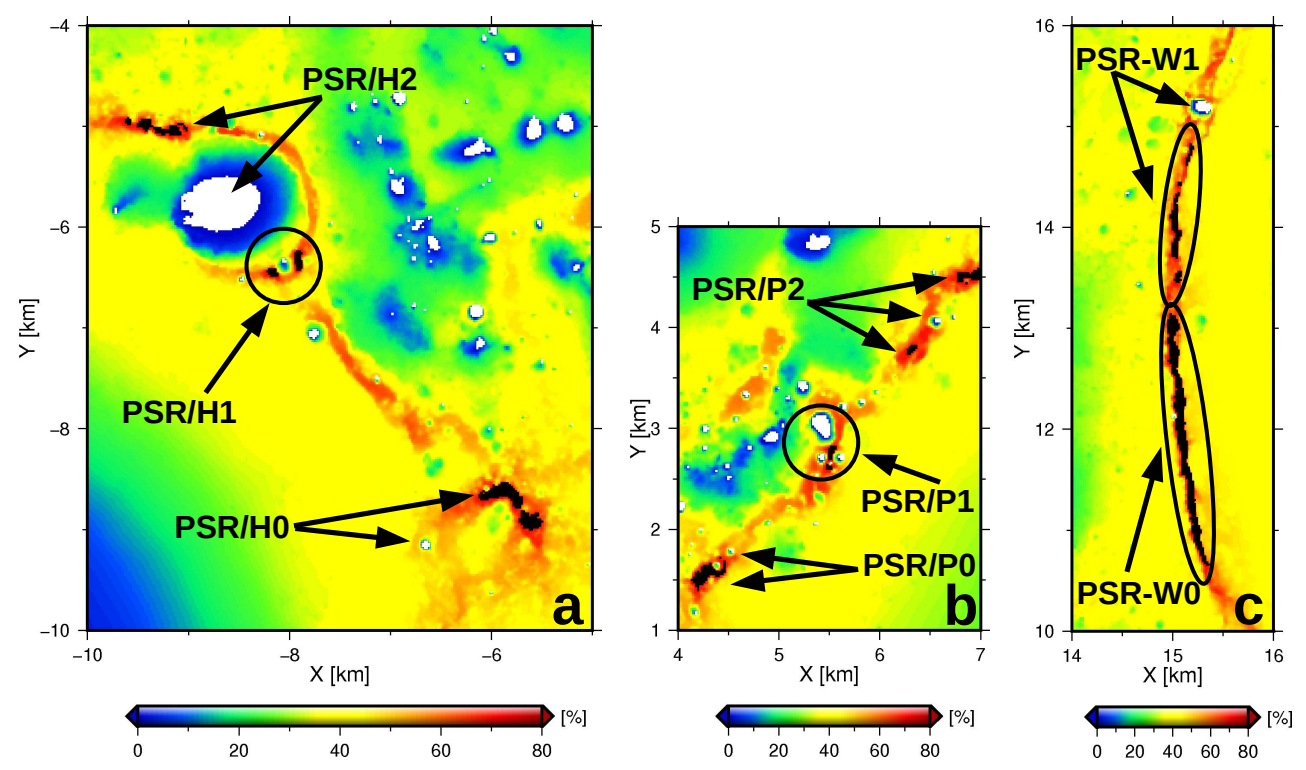

Figure 5: The three identified north-polar areas receiving the highest accumulated illumination (shown from 0-80\%) at two meters above ground over the chosen twenty-year period. The areas of high average illumination $(>70 \%)$ are shown as black pixels and PSRs are shown as white pixels. (a) At Hinshelwood crater, three clusters of high average illumination are found at $\mathrm{H} 0$, H1, H2 with nearby PSRs. (b) Three clusters P0, P1, P2 are found at Peary crater. The chosen PSR at P1 is composed of three PSRs surrounding P1. (c) At Whipple crater two clusters with PSRs nearby are found. Map axis units are in kilometers and map is displayed in gnomonic projection.

$78.8 \%$. Note, that despite the fact that the location with the highest average illumination on Shackleton crater rim is found at cluster S0, the location with the longest solar visibility is found at $\mathrm{S} 1$. At $\mathrm{S} 0$ we find a continuous cluster of $21,600 \mathrm{~m}^{2}$ next to two PSRs of a total of $58,400 \mathrm{~m}^{2}$ in 370 meters distance. The average slope within the PSR is $8.6^{\circ}$ with a maximum slope of $17.0^{\circ}$. Along the rim towards the south pole we identified $\mathrm{S} 1$ with almost continuous $120,400 \mathrm{~m}^{2}$, of which parts are enclosing a PSR of $4,400 \mathrm{~m}^{2}$ in just thirty meters distance. Here the average slope is $7.9^{\circ}$ with a maximum slope within the PSR of $11.1^{\circ}$. The last cluster of $\mathrm{S} 2$ is composed of a total area of 31,200 $\mathrm{m}^{2}$ which is rather remotely connected. It adjoins the nearby 1,200 $\mathrm{m}^{2}$ PSR in eighty meters distance, where an average slope of $3.6^{\circ}$ and a maximum slope of $6^{\circ}$ can be found.

At the proposed south-polar sites all slopes are below the maximum angle of $21.8^{\circ}$ inclination that a lunar rover can pass (Sheng and Wei-Dong, 2014). The results for the south-polar sites are summarized in Table 3. 

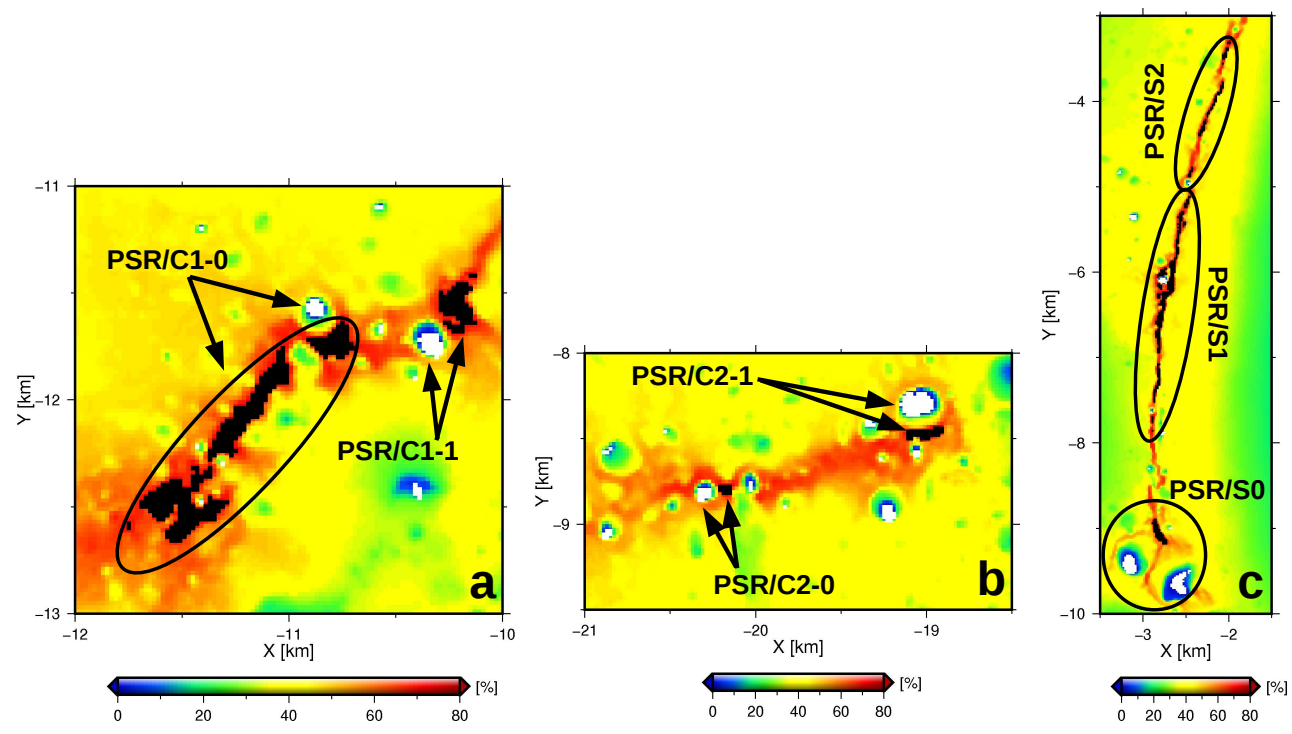

Figure 6: The three identified south-polar areas receiving the highest accumulated illumination (shown from 0-80\%) at two meters above ground over the chosen twenty-year period. The areas of high average illumination $(>70 \%)$ are shown as black pixels and PSRs are shown as white pixels. (a) $\mathrm{C} 1$ at Connecting Ridge incorporates two clusters of high average illumination $\mathrm{C} 1$ 0 and C1-1. (b) C2 also contains two clusters C2-0 and C2-1. (c) At Shackleton crater rim three clusters with PSRs nearby can be identified. Map axis units are in kilometers and map is displayed in gnomonic projection. 


\begin{tabular}{|r|r|r|r|r|r|r|}
\hline & $\begin{array}{r}\text { Maximum } \\
\text { Average } \\
\text { Illumination } \\
{[\%]}\end{array}$ & $\begin{array}{r}\text { Maximum } \\
\text { Solar } \\
\text { Visibility } \\
{[\%]}\end{array}$ & $\begin{array}{r}\text { Size } \\
\text { of } \\
\text { Cluster } \\
{\left[\mathrm{m}^{2}\right]}\end{array}$ & $\begin{array}{r}\text { Size } \\
\text { of } \\
\text { PSR } \\
{\left[\mathrm{m}^{2}\right]}\end{array}$ & $\begin{array}{r}\text { Distance } \\
\text { to } \\
\text { PSR } \\
{[\mathrm{m}]}\end{array}$ & $\begin{array}{r}\text { Maximum } \\
\text { Slope } \\
\text { PSR } \\
{\left[{ }^{\circ}\right]}\end{array}$ \\
\hline North pole & \multicolumn{7}{|c|}{} \\
\hline Hinshelwood, H0 & 81.5 & 89.3 & 82,400 & 5,200 & 680 & 11.9 \\
Hinshelwood, H1 & 74.1 & 81.1 & 19,600 & 1,200 & 100 & 3.5 \\
Hinshelwood, H2 & 75.0 & 82.3 & 36,800 & 320,400 & 520 & 22.1 \\
\hline Peary, P0 & 77.1 & 85.1 & 36,000 & 1,200 & 130 & 5.5 \\
Peary, P1 & 72.7 & 80.3 & 10,400 & 34,000 & 60 & 15.1 \\
Peary, P2 & 74.7 & 82.2 & 26,800 & 3,600 & 310 & 9.7 \\
\hline Whipple, W0 & 85.1 & 90.8 & 175,600 & 1,200 & 40 & 7.0 \\
Whipple, W1 & 76.9 & 83.1 & 48,800 & 14,400 & 350 & 20.0 \\
\hline South pole & \multicolumn{7}{|c|}{} & & \\
\hline Connecting Ridge, C1-0 & 88.0 & 92.1 & 135,200 & 5,200 & 100 & 12.8 \\
Connecting Ridge, C1-1 & 81.3 & 86.0 & 26,800 & 9,600 & 70 & 13.7 \\
\hline Connecting Ridge, C2-0 & 71.6 & 75.1 & 4,400 & 5,600 & 60 & 11.5 \\
Connecting Ridge, C2-1 & 78.1 & 82.0 & 11,600 & 22,800 & 80 & 17.1 \\
\hline Shackleton, S0 & 85.5 & 89.4 & 21,600 & 58,400 & 370 & 17.0 \\
Shackleton, S1 & 84.3 & 89.8 & 120,400 & 4,400 & 30 & 11.1 \\
Shackleton, S2 & 73.9 & 78.8 & 31,200 & 1,200 & 80 & 6.0 \\
\hline
\end{tabular}

Table 3: List of clusters at Hinshelwood, Peary and Whipple craters with favorable illumination conditions at two meters above ground (column one). Shown are the maximum average illumination and solar visibility of each cluster in percentage (columns two and three), the size of the cluster and the PSR in square-meters (columns four and five), and the closest distance between the cluster and the PSR in meters (column six) and the maximum encountered slope within the PSR in degrees (column seven). 


\section{Conclusion}

A north polar LOLA DTM was generated and a previously created south polar LOLA DTM was updated for this work. Based on illumination simulations on these LOLA DTMs we identified a total of six potential exploration sites near both lunar poles characterized by long illumination and proximity to a PSR. On the north pole we identified the three sites along the crater rims of Hinshelwood, Peary and Whipple crater and at the south pole two sites along Connecting Ridge and one on the rim of Shackleton crater. We found that these sites are typically illuminated for about $90 \%$ of the time. The almost constant illumination at the identified sites would guarantee longer mission durations on the lunar surface than previous endeavors. In general the long, obviously dark and hence extremely cold lunar night is the main problem for the electronics carried by surface mission. At the identified sites the lunar night is short and vital equipment can either be heated electronically or by the Sun. Maximum times in shadow of less than three and up to eight days were calculated for these sites, which is only $20-50 \%$ of the lunar night. Encountered slopes at the proposed sites typically do not exceed the maximum angle of inclination for a lunar rover and hence rovers could repeatedly traverse between PSRs and illuminated spots.

For a robotic mission towards the north pole, Whipple crater represents the most favorable landing site because the largest area of high average illumination and also the highest average illumination are found here. The two clusters W0 and W1 are almost connected and would allow for traversing along both clusters to maximize time in the Sun, similar to the work of Speyerer et al. (2016) at Shackleton crater. Although the identified nearby PSRs are small and might not harbor surficial water-ice due to stray light from very close sunlit areas, detection of ice might be possible within the upper layer of regolith (few centimeters to decimeters), which would help in understanding subsurface migration of water-ice and cold traps in general (Schorghofer and Taylor, 2007). Also, the large PSR inside Whipple crater is only few kilometers away and could either be investigated remotely or in-situ. At Hinshelwood slightly lower illumination levels and briefer durations in the Sun are found with an overall smaller area of high average illumination than at Whipple crater but with a much larger nearby PSR. The large PSR inside Hinshelwood is also within reach. The clusters at Peary crater are least favorable with regard to size and illumination level.

Near the south pole, Shackleton crater is the analog site to Whipple crater on the north pole. A similar distribution, size and illumination of the clusters along the rim can be found. Although the distance to the nearby PSRs and to the large PSR inside Shackleton is comparable to that of Whipple, the total size of the PSR is 
much larger at the south pole. Nevertheless, the clusters at Connecting Ridge C1 have similar attributes with even slightly longer and higher illumination levels. The shape of the area of high average illumination makes $\mathrm{C} 1$ more accessible for exploration. The second site on Connecting Ridge, C2, is the least favorable regarding illumination and areal extent.

Acknowledgments. P. Gläser was funded by a Grant of the German Research Foundation (FOR 1503, GL 865/2-1). J. Oberst gratefully acknowledges being hosted by MIIGAiK and supported by the Russian Science Foundation under project 14-22-00197. We wish to thank the LOLA and LROC Science Team for releasing such great data products. The authors would also like to acknowledge the helpful input of an anonymous reviewer, which significantly improved the final version of this manuscript.

Arvidson, R.E., Squyres, S.W., Gellert, R., Athena Science Team, 2015. Recent Results from the Opportunity Rover's Exploration of Endeavour Crater, Mars, in: Lunar and Planetary Science Conference, p. 1118.

Bussey, D.B.J., McGovern, J.A., Spudis, P.D., Neish, C.D., Noda, H., Ishihara, Y., Sørensen, S.A., 2010. Illumination conditions of the south pole of the Moon derived using Kaguya topography. Icarus 208, 558-564.

Bussey, D.B.J., Spudis, P.D., Robinson, M.S., 1999. Illumination conditions at the lunar south pole. Geophysical Research Letters 26, 1187-1190.

Cohen, B.A., Hayne, P.O., Greenhagen, B.T., Paige, D.A., 2015. Lunar Flashlight: Exploration and Science at the Moon with a $6 \mathrm{U}$ CubeSat, in: Annual Meeting of the Lunar Exploration Analysis Group, p. 2008.

De Rosa, D., Bussey, B., Cahill, J.T., Lutz, T., Crawford, I.A., Hackwill, T., van Gasselt, S., Neukum, G., Witte, L., McGovern, A., Grindrod, P.M., Carpenter, J.D., 2012. Characterisation of potential landing sites for the European Space Agency's Lunar Lander project. Planetary and Space Science 74, 224-246.

Feldman, W.C., Maurice, S., Lawrence, D.J., Little, R.C., Lawson, S.L., Gasnault, O., Wiens, R.C., Barraclough, B.L., Elphic, R.C., Prettyman, T.H., Steinberg, J.T., Binder, A.B., 2001. Evidence for water ice near the lunar poles. Journal of Geophysical Research 106, 2323123252.

Gläser, P., Haase, I., Oberst, J., Neumann, G.A., 2013. Co-registration of laser altimeter tracks with digital terrain models and applications in planetary science. Planetary and Space Science 89, 111-117.

Gläser, P., Scholten, F., De Rosa, D., Marco Figuera, R., Oberst, J., Mazarico, E., Neumann, G.A., Robinson, M.S., 2014. Illumination conditions at the lunar south pole using high resolution Digital Terrain Models from LOLA. Icarus 243, 78-90.

Margot, J.L., Campbell, D.B., Jurgens, R.F., Slade, M.A., 1999. Topography of the lunar poles from radar interferometry: A survey of cold trap locations. Science 284, 1658.

Mazarico, E., Neumann, G.A., Smith, D.E., Zuber, M.T., Torrence, M.H., 2011. Illumination conditions of the lunar polar regions using LOLA topography. Icarus 211, 1066-1081. 
McGovern, J.A., Bussey, D.B., Greenhagen, B.T., Paige, D.A., Cahill, J.T.S., Spudis, P.D., 2013. Mapping and characterization of non-polar permanent shadows on the lunar surface. Icarus 223, 566-581.

Miller, R.S., Lawrence, D.J., Hurley, D.M., 2014. Identification of surface hydrogen enhancements within the Moon's Shackleton crater. Icarus 233, 229-232.

Mitrofanov, I., Litvak, M., Sanin, A., Malakhov, A., Golovin, D., Boynton, W., Droege, G., Chin, G., Evans, L., Harshman, K., Fedosov, F., Garvin, J., Kozyrev, A., McClanahan, T., Milikh, G., Mokrousov, M., Starr, R., Sagdeev, R., Shevchenko, V., Shvetsov, V., Tret'yakov, V., Trombka, J., Varenikov, A., Vostrukhin, A., 2012. Testing polar spots of water-rich permafrost on the Moon: LEND observations onboard LRO. Journal of Geophysical Research (Planets) 117,0 .

Noda, H., Araki, H., Goossens, S., Ishihara, Y., Matsumoto, K., Tazawa, S., Kawano, N., Sasaki, S., 2008. Illumination conditions at the lunar polar regions by KAGUYA(SELENE) laser altimeter. Geophysical Research Letters 35, 24203.

Paige, D.A., Siegler, M.A., Zhang, J.A., Hayne, P.O., Foote, E.J., Bennett, K.A., Vasavada, A.R., Greenhagen, B.T., Schofield, J.T., McCleese, D.J., Foote, M.C., DeJong, E., Bills, B.G., Hartford, W., Murray, B.C., Allen, C.C., Snook, K., Soderblom, L.A., Calcutt, S., Taylor, F.W., Bowles, N.E., Bandfield, J.L., Elphic, R., Ghent, R., Glotch, T.D., Wyatt, M.B., Lucey, P.G., 2010. Diviner Lunar Radiometer Observations of Cold Traps in the Moon's South Polar Region. Science 330, 479.

Rosenburg, M.A., Aharonson, O., Head, J.W., Kreslavsky, M.A., Mazarico, E., Neumann, G.A., Smith, D.E., Torrence, M.H., Zuber, M.T., 2011. Global surface slopes and roughness of the Moon from the Lunar Orbiter Laser Altimeter. Journal of Geophysical Research (Planets) 116, E02001.

Schorghofer, N., Taylor, G.J., 2007. Subsurface migration of $\mathrm{H}_{2} \mathrm{O}$ at lunar cold traps. Journal of Geophysical Research (Planets) 112, E02010.

Sheng, Y., Wei-Dong, H., 2014. Motion control strategy and simulation for slope travel of a lunar rover. Proceedings of 2014 International Conference on Mechanical Design, Manufacture and Automation Engineering(MDMAE 2014), 244-251.

Smith, D.E., Zuber, M.T., Jackson, G.B., Cavanaugh, J.F., Neumann, G.A., Riris, H., Sun, X., Zellar, R.S., Coltharp, C., Connelly, J., Katz, R.B., Kleyner, I., Liiva, P., Matuszeski, A., Mazarico, E.M., McGarry, J.F., Novo-Gradac, A.M., Ott, M.N., Peters, C., Ramos-Izquierdo, L.A., Ramsey, L., Rowlands, D.D., Schmidt, S., Scott, V.S., Shaw, G.B., Smith, J.C., Swinski, J.P., Torrence, M.H., Unger, G., Yu, A.W., Zagwodzki, T.W., 2010. The Lunar Orbiter Laser Altimeter Investigation on the Lunar Reconnaissance Orbiter Mission. Space Science Reviews 150, 209-241.

Smith, D.E., Zuber, M.T., Neumann, G.A., Mazarico, E., Lemoine, F.G., Head, III, J.W., Lucey, P.G., Aharonson, O., Robinson, M.S., Sun, X., Torrence, M.H., Barker, M.K., Oberst, J., Duxbury, T.C., Mao, D., Barnouin, O.S., Jha, K., Rowlands, D.D., Goossens, S., Baker, D., Bauer, S., Gläser, P., Lemelin, M., Rosenburg, M., Sori, M.M., Whitten, J., Mcclanahan, T., 2017. Summary of the results from the lunar orbiter laser altimeter after seven years in lunar orbit. Icarus 283, 70-91.

Smith, W.H.F., Wessel, P., $1990 . \quad$ Gridding with continuous curvature splines in tension. Geophysics 55, 293-305. http://geophysics.geoscienceworld.org/content/55/3/293.full.pdf.

Speyerer, E.J., Lawrence, S.J., Stopar, J.D., Gläser, P., Robinson, M.S., Jolliff, B.L., 2016. Op- 
timized traverse planning for future polar prospectors based on lunar topography. Icarus 273, 337-345.

Speyerer, E.J., Robinson, M.S., 2013. Persistently illuminated regions at the lunar poles: Ideal sites for future exploration. Icarus 222, 122-136.

Spudis, P.D., Bussey, D.B.J., Baloga, S.M., Cahill, J.T.S., Glaze, L.S., Patterson, G.W., Raney, R.K., Thompson, T.W., Thomson, B.J., Ustinov, E.A., 2013. Evidence for water ice on the moon: Results for anomalous polar craters from the LRO Mini-RF imaging radar. Journal of Geophysical Research (Planets) 118, 2016-2029.

Vasavada, A.R., Paige, D.A., Wood, S.E., 1999. Near-Surface Temperatures on Mercury and the Moon and the Stability of Polar Ice Deposits. Icarus 141, 179-193.

Watson, K., Murray, B., Brown, H., 1961. On the possible presence of ice on the Moon. Journal of Geophysical Research 66, 1598-1600.

Wessel, P., Smith, W.H.F., Scharroo, R., Luis, J., Wobbe, F., 2013. Generic mapping tools: Improved version released. Eos, Transactions American Geophysical Union 94, 409-410. 